\title{
Innovative Technologies of Healthcare
}

\author{
Serik Smagulov, \\ Viktoriya Smagulova MD
}

\begin{abstract}
This article discusses the possibilities of modernizing the healthcare system using the latest technological developments to improve the quality of the healthcare system, the quality of patient care and the creation of new opportunities for patients using Digital Transformation Technology. The purpose of the study was to understand the growing role of health digitization, based on the experience of European countries, to identify the problems faced by IT health professionals and how these problems affect the system of health care and the possibility of improving the health system. We looked at ASI technology, Chatbot, the NETSCOUT platform, and other major innovations in digital health care technology. The result of the considered modernization due to the introduction of new programs is to reduce costs and improve the quality of care and patient care.
\end{abstract}

Keywords: Digital Transformation, digitalization, personal data, Internet of Things, big data, personal health records, personal health information, diagnostic tools, healthcare, transformation, chatbot, HIPAA, NETSCOUT

In the field of health, there has been an evolution of digital technology, the impact of which is truly enormous. It should be noted that digital transformation is stimulated by the use of technologies such as mobility, Internet of Things (IoT), Web 3.0 platforms, various unified communications (UC), etc. In some developed countries, digital technologies can provide uninterrupted data flow, ranging from medical records and systems to interactions between doctors and patients at any time and from any place.

As for the future of such a digital transformation, each state seeking to modernize the health care system with the help of digital transformation technology sets the ultimate goal of reducing costs and improving the quality of patient care. Medical organizations which use mobility, Big Data, UC and IoT strive to expand the entire chain of medical services from diagnostics to subsequent care, improving the relationship between patients, doctors and insurance companies. With the use of digital technology, all processes are becoming more automated, efficient and mobile.

There is currently a transition from the use of physical assets to digital assets. But there are still practical problems for achieving the correct and accurate functioning of the entire health care system in introducing modern digital technologies. 
The introduction of digital technology has an impact on how health care workers diagnose, monitor and monitor the health of patients. Digital services include electronic medical records and enterprise resource planning systems. These digital services are directly related to the provision of medical services (from portable diagnostic tools and crowdsourcing to the development of serious genomic applications, for example, to decoding DNA on a portable device).

The speed of digital transformation and its countless number of digital services contribute to the development of healthcare, but there are also pitfalls here - ranging from the integration of infrastructure and applications to the timely and high-quality provision of services. It is essential how the requirements and expectations of patients are satisfied.

IT professionals in the field of healthcare often face toeing some problems. These problems include such issues as maintaining a developing service delivery infrastructure, identifying service performance problems, ensuring the availability of clinical applications $24 / 7 / 365$.

Empowering patients leads to significant changes in health care. But confidence in providing the best patient care is possible only with timely access to information. That is why continuous data monitoring based on traffic and real-time analytics is so crucial for determining the best treatment options and achieving successful results for patients.

One of the most critical components for healthcare providers is the ongoing monitoring of EHR transactions.

Health care providers must be able to track Health Level-7 protocol (HL7) transactions, as well as measure application transaction performance based on HL7, detect related error messages, and decode HL7 data fields [1].

The NETSCOUT nGeniusONE Service Assurance platform with its patented Adaptive Service Intelligence (ASI) technology is used in healthcare of many countries. The main functionality of NETSCOUT nGeniusONE allows you to determine the performance problems of services accurately, because it combines continuous monitoring and multi-level analytical capabilities in real time, providing an integrated solution for managing performance in the most complex and demanding IT service environments [2]. 
ASI technology continuously converts large amounts of traffic-based data into structured metadata optimized for real-time analytics platforms, which, in turn, are capable of supporting the evolving network infrastructure of service provision, while ensuring roundthe-clock accessibility for patients.

The NETSCOUT platform allows network and application teams to quickly identify and sort service performance problems and reduce risks for both the organization and the patients it ultimately serves [3].

Another new trend in the digital health industry is chatbots, which act as digital assistants for doctors.

As in any other industry, cost savings are also a significant concern for the healthcare industry. Chatbot technology helps to solve common medical inquiries at an affordable price using messaging systems and AI-enabled voice systems. Modern chatbots are designed to study the interaction with the patient and assuming the role of the general practitioner. Some chat robots, such as Woebot, now also have the opportunity to act as digital therapists. Smart bots serve as digital assistants for doctors in tracking contacts and arranging meetings with patients. On the other hand, chatbots also make life easier for patients by providing timely recipes, conducting laboratory tests and making the billing process easy.

Even though chatbots technology is still at an early stage of development, research firm Grand View Research has calculated that the global chat bots market will reach \$ 1.23 billion by 2025 . The United States and the cumulative annual growth rate (CAGR) will be $24.3 \%[4]$.

In the current digital healthcare industry, medical chatbots can be divided into two categories: the first is patient-only applications that help patients keep track of health data; and, secondly, clinician-patient applications that serve as a link between the two groups for diagnosis and treatment.

It is worth noting the growing role of artificial intelligence (AI) in health care. This is another emerging digital healthcare technology. With the help of data analysis, physicians 
can now explore various approaches to treatment. Scanning the body with artificial intelligence reveals many chronic severe diseases at an early stage.

Pharmaceutical companies are introducing machine learning algorithms to study chemical and biological interactions to develop new drugs, replacing an earlier clinical trial process that used to cost billions of dollars and take more than ten years [5].

Another critical point in the digitalization of healthcare is the introduction of cloud services. The main reason for the rapid adoption of cloud computing in the health sector is that only cloud solutions provide health care workers and patients with the necessary access in accordance with the Health Insurance Mobility and Accountability Act (HIPAA), which sets standards for data protection [6], a recent CIF study found that $98 \%$ of companies have never experienced security breaches when using a cloud service. World experts predict that more than $50 \%$ of the healthcare industry enterprises that are on the path to digital transformation will rely on at least one public cloud platform.

More and more healthcare providers, payers, and IT professionals are using cloudbased pay-per-use cloud services to process, store, and transfer sensitive medical information.

For example, Amazon - AWS allows organizations covered by the United States Healthcare Authority Data Transfer and Protection Act 1996 (HIPAA) 1996 and their business partners to use the AWS secure environment to process, maintain and store sensitive medical information.

Digital transformation is rapidly transforming the healthcare industry. From the material examined by us, it can be concluded that patients have moved from passive recipients of medical care to active consumers. As a result of this transition and the policies of the governments of countries, health care providers should improve patient care and improve the quality of care. This requires the adoption of a large number of custom applications.

Summarizing, it can be noted that numerous digital solutions, such as, for example, the NETSCOUT considered in this article, provide uninterrupted service performance. This allows providing high-quality services to patients while optimizing costs. 


\section{References}

1. An HL7 (Health Level Seven) overview// Journal of AHIMA / American Health Information Management Association 70(7):32-4; quiz 35-6 · July 1999

2. Eileen Singh. NETSCOUT: Manage Unified Communications Efficiently// https://unified-communications.cioapplications.com/vendor/netscout-nasdaq-ntctmanage-unified-communications-efficiently-cid-1116-mid-60.html

3. Jack W. Plunkett. Plunkett's E-Commerce \& Internet Business Almanac. Plunkett Research, Ltd., 2009

4. Chatbot Market Size To Reach \$1.25 Billion By 2025 |CAGR: 24.3\% // https://www.grandviewresearch.com/press-release/global-chatbot-market

5. D. DouglasMillerMD, CM FACPEric W.BrownPhD Artificial Intelligence in Medical Practice: The Question to the Answer?// The American Journal of Medicine Volume 131, Issue 2, February 2018, Pages 129-133

6. Juliana De Groot. What is HIPAA Compliance? 2019 HIPAA Requirements // https://digitalguardian.com/blog/ 